\title{
Identification of new Bremia lactucae races in lettuce in São Paulo state
}

\author{
Renata Castoldi'; Hamilton César de O Charlo²; Tagli Dalpian³; Danilo M Melo'; Adelita P Botelho'; \\ Leila T Braz ${ }^{1}$ \\ ${ }^{1}$ UNESP-FCAV, Depto Produção Vegetal, Rod. Prof. Paulo Donato Castellane s/n, 14884-900 Jaboticabal-SP; rcastoldi@gmail.com;

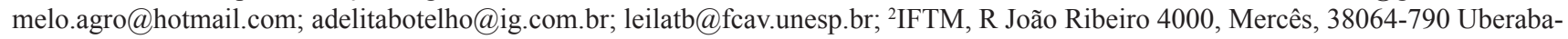 \\ MG; hamiltoncharlo@iftm.edu.br; ${ }^{3}$ CATI, Casa da Agricultura de Ibirá, Av Gabriel Sanches 725, 15860-000 Ibirá-SP; tagli.dalpian@cati. \\ sp.gov.br
}

\begin{abstract}
Lettuce is the most consumed leafy vegetable in Brazil. However, its production has faced increasing difficulties mainly due to the infestation of producing areas with Bremia lactucae, the causing agent of downy mildew, the worst disease in lettuce. The aim of this study was to identify $B$. lactucae races occurring in lettuce producing areas of São Paulo state, during 2008 and 2009. Lettuce leaf samples containing $B$. lactucae sporangia were collected in the major lettuce producing areas of São Paulo. Each sample was considered an isolate. After the multiplication of sporangia in the susceptible cultivar Solaris, with subsequent inoculation in the differentiating cultivars, the evaluations were performed on the same day of the first sporulation in the susceptible cultivar 'Cobham Green (DM 0)'. In 2008 one new B. lactucae code was identified. SPB1:05 was the denomination proposed to it. In 2009 one another B. lactucae code was identified, that is, $63 / 63 / 02 / 00$, whose denomination was proposed to be SPB1:06.
\end{abstract}

Keywords: Lactuca sativa L, genes DM, downy mildew, resistance.

\section{RESUMO}

Identificação de novas raças de Bremia lactucae em alface no Estado de São Paulo

A alface é o grupo de hortaliças folhosas de maior consumo no Brasil. No entanto a dificuldade em produzi-la vem aumentando, principalmente pela infestação das áreas de produção por Bremia lactucae, agente causador do míldio, principal doença da alface. $\mathrm{O}$ objetivo deste trabalho foi identificar as raças de B. lactucae que ocorreram nos municípios produtores de alface do Estado de São Paulo nos anos de 2008 e 2009. Para isso, coletaram-se folhas com esporângios de $B$. lactucae em municípios produtores de alface, sendo que cada amostra foi considerada um isolado. Após a multiplicação dos esporângios na cultivar suscetível Solaris, com posterior inoculação nas diferenciadoras, realizaram-se as avaliações, no mesmo dia do aparecimento da primeira esporulação na cultivar suscetível 'Cobham Green (DM 0)'. No ano 2008 identificou-se um novo código de $B$. lactucae em alface, à qual se propôs a denominação de SPB1:05. No ano 2009, outro código de B. lactucae foi identificado (63/63/02/00), ao qual se propôs a denominação de SPBl:06.

Palavras-chave: Lactuca sativa, genes DM, míldio, resistência.

(Recebido para publicação em 25 de abril de 2011; aceito em 14 de março de 2012) (Received on April 25, 2011; accepted on March 14, 2012)

Lor ettuce (Lactuca sativa), a member of the Asteraceae family, is a plant originating from Asia that was introduced in Brazil in the XVI century (Oliveira et al., 2004). Among the leafy vegetables, lettuce is the most widely consumed in Brazil due to the fact it is available year-round. In the state of São Paulo, between January and July 2010, 29,297 tons of lettuce were commercialized, $47.34 \%$ of them formed by cultivars of the crispy group (Agrianual, 2011).

The major lettuce producers are found in the 'green belt' of São Paulo city, such as Mogi das Cruzes, where is located the greatest lettuce cultivation area (3.4 thousand ha) (IEA, 2010). Other small areas are also found all over São Paulo state, mainly near the major cities. The lettuce producing areas have steadily increased in number during the last years.

The increment in lettuce production also caused increased production difficulties, even with more adequate environmental conditions such as autumn and winter seasons. There occur an increase of infestation of these areas by Bremia lactucae, the causing agent of downy mildew, which is considered the worst problem affecting lettuce production (Yuri et al., 2004).

The species B. lactucae (Oomycetes) is a biotrophic parasite found in the living cells of the host plant. This parasite survives only on living lettuce plants (Michelmore \& Ingram, 1982). The lesions caused by mildew are of light green color or slightly chlorotic which become yellow or necrotic after the pathogen sporulation (Van Bruggen $\&$ Scherm, 1997). The disease is usually controlled by the application of systemic fungicides, but the continued use of these products, as well as the use of vertical resistance, or environmental modification, though may result in the selection of resistant pathogens which may eventually end up by developing in new races capable of establishing themselves even in resistant cultivars. So the most economical way of controlling the disease consists in the use of resistant cultivars.

A previous study reported by Braz et al. (2007), undertaken during 2003 and 2004 in the state of São Paulo, showed the race SPBl:01 to be the predominant one. In the same study, the authors 
verified the lettuce genes R-17, R-18, and R-38 to be capable of conferring resistance to the race $\mathrm{SPB1}: 01$. More recently, a study carried out in 2006 and 2007 showed the existence of three new races, $\mathrm{SPBl}: 02, \mathrm{SPBl}: 03$, and $\mathrm{SPBl}: 04$, to which the lettuce genes R-17, R-18, $\mathrm{R}-37$, and -38 were verified to confer resistance (Souza et al., 2011).

In California, USA, four main $B$. lactucae pathotypes were found which were identified by the determination of their virulence with the help of differentiating cultivars (Schettini et al., 1991).

According to the Plantum (2010), the International Bremia Evaluation Board (IBEB) evaluated several lettuce isolates during 2009 and showed the existence of 27 races of $B$. lactucae in Europe, and the majority of the foci were caused by new races whose importance was restricted to the local where they were found.

The IBEB notes that although the seed companies submit seeds with resistance to the races $\mathrm{Bl}: 1$ to $\mathrm{Bl}: 27$, this is not totally secure against the pathogen, because it provides to the producer only temporary protection, as through mutation, the combination of several genes existing in the same cultivar may be broken (Plantum NL, 2010).

Thus, the aim of this study was to identify $B$. lactucae races occurring in lettuce producing areas of São Paulo state during 2008 and 2009 aiming to support the lettuce breeding program in progress at the Department of Plant Production, a unity of the Paulista State University in its campus of Jaboticabal.

\section{MATERIAL AND METHODS}

During August, September, and October of 2008, and August and September of 2009, lettuce leaves with sporangia of B. lactucae were sampled from producing areas located in 17 counties of São Paulo state, namely Ribeirão Preto, Jaboticabal, Pirangi, Catanduva, São José do Rio Preto, Atibaia, Salesópolis, Biritiba Mirim, Mogi das Cruzes, Itapira, Mogi Mirim, Cândido Mota, Presidente Prudente,
Echaporã, Marilia, Botucatu, and Bauru. Each sample was considered an isolate and the number of collected isolates during the years of 2008 and 2009, were 35 and 49 were respectively.

The samples were put in plastic bags, identified and stored in thermos to be transported to the Plant Breeding Laboratory of the Department of Plant Production, where the sporangia were multiplied in plants of the 'Solaris' cultivar, a susceptible one to obtain the necessary amount of sporangia for the differentiation test.

The next phase consisted in the sowing of seeds of the 21 differentiating cultivars in $11 \times 11 \times 3.5 \mathrm{~cm}$ plastic boxes lined with paper employed for germination tests. This paper was wet and divided in four equal parts. In each one of these parts, approximately 40 seeds of each differentiating cultivar were sown and maintained for 15 days at a temperature of $13^{\circ} \mathrm{C}$ and a photoperiod of 12 hours, in a seed germinator. After that period, the collected isolates were inoculated according to technique of Illot et al. (1987). The sporangia used in the procedure were obtained by washing infected tissues of the host plants after being stirred in distilled water. A suspension containing $5 \times 10^{4}$ sporangia/mL was used to be sprayed on the seedlings.

Following the inoculation, the boxes were taken back to the germinator and kept at $13^{\circ} \mathrm{C}$ in darkness for the first 6 hours. After that period, the photoperiod was adjusted to 12 hours.

Evaluations were daily and when the first sporulation was detected in the susceptible cultivar 'Cobham Green (DM 0)', the differentiating cultivars were individually evaluated for the occurrence of sporulation and necrosis, according to methodology proposed by Van Der Arend et al. (2003). This is a procedure by which the signs $+,(+),-$, or (-) are ascribed to lettuce seedlings according to the following criteria: + when more than $80 \%$ of the seedlings show sporulating lesions; $(+)$ when more than $80 \%$ of the seedlings show necrotic points and several sporulating lesions; - when less than 5\% of the seedlings show sporulating lesions; and (-) when the seedlings show necrotic points and few sporulating lesions.

All the materials showing susceptibility (with positive sign), to a given isolate had their values added within each group (from 1 to 4), resulting in four distinct numbers, each one separated by a bar, that identifies the different groups. This numerical sequence was then called "Sextet" Code.

\section{RESULTS AND DISCUSSION}

In 2008, the results showed the existence of four codes of B. lactucae in the lettuce producing regions of São Paulo state, namely 63/63/03/00,63/63/33/00, $63 / 63 / 51 / 00$, and $63 / 63 / 19 / 00$. The sextet code $63 / 63 / 03 / 00$ was the most frequent one $(48.57 \%$ of the isolates) (Table 1).

The sextet code 63/63/03/00 was reported by Souza et al. (2011) too, who found it in 2006 in a low level of frequency but enough to give origin to a new race named SPBl:04. Therefore, this race should be a focal point in lettuce breeding programs, since its virulence genes were capable of breaking down the resistance of lettuce genotypes developed by Souza, which is resistant only to the race $\mathrm{SPB1}: 01$. This race was disseminated very efficiently since 2006, when it was first reported in the county of Bauru. However, two years late, it was found in seven other municipalities of São Paulo state in addition to Bauru. This fact justifies the necessity of a constant monitoring of the lettuce producing regions as part of a strategy for the control of the disease by means of resistant genotypes.

The code 63/63/33/00 was detected in the majority of the lettuce producing regions and may thus be considered as a new race to which the denomination SPBl:05 was proposed by Souza et al. (2011). The difference between this race and those already found in São Paulo state (SPB1:01, SPB1:02, SPB1:03, and $\mathrm{SPBl}: 04)$ consists in the resistance of the DM-16 gene in the formation of the sextet code. But, the use of that gene in lettuce genetic improvement programs is not recommended due to its susceptibility to the codes $63 / 63 / 51 / 00$, $63 / 63 / 19 / 00$, and 63/63/03/00. 
Table 1. Resistance/susceptibility response of lettuce differentiating cultivars to Bremia lactucae isolates collected during 2008 in the major lettuce producing regions of São Paulo state (resposta de resistência/suscetibilidade de cultivares diferenciadoras à Bremia lactucae dos isolados coletados durante o ano de 2008, nos principais municípios produtores de alface do estado de São Paulo). Jaboticabal, UNESP, 2011.

\begin{tabular}{|c|c|c|c|c|c|c|c|c|c|c|c|c|c|c|c|c|c|c|c|c|c|c|}
\hline 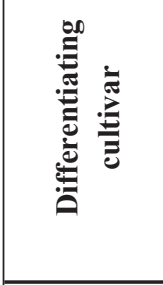 & 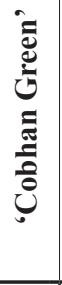 & 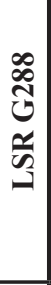 & 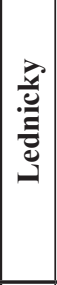 & $\begin{array}{l}\tilde{a} \\
\tilde{a} \\
己 \\
己\end{array}$ & 氣 & 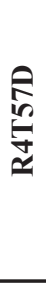 & 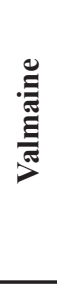 & 兽 & 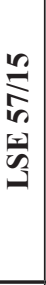 & 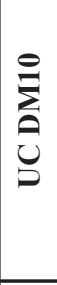 & 苞 & 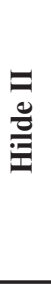 & 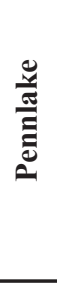 & 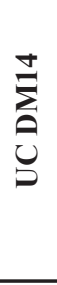 & 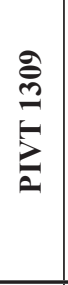 & نِ & $\frac{\widetilde{\sigma}}{\mathfrak{\sigma}}$ & $\frac{\stackrel{0}{\pi}}{\frac{\pi}{0}}$ & $\underset{\mathrm{Z}}{\stackrel{\sigma}{\Xi}}$ & 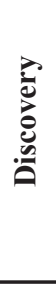 & 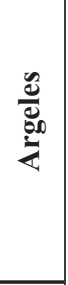 & 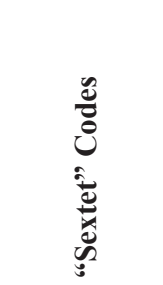 \\
\hline Genes DM & - & - & 1 & 2 & 3 & 4 & $5 / 8$ & 6 & 7 & 10 & 11 & 12 & 13 & 14 & 15 & 16 & 17 & 18 & 36 & 37 & 38 & \\
\hline Sextet No. & - & - & 1 & 2 & 3 & 4 & 5 & 6 & 7 & 8 & 9 & 10 & 11 & 12 & 13 & 14 & 15 & 16 & 17 & 18 & 19 & \\
\hline Values & - & - & 1 & 2 & 4 & 8 & 16 & 32 & 1 & 2 & 4 & 8 & 16 & 32 & 1 & 2 & 4 & 8 & 16 & 32 & 1 & \\
\hline \multirow[t]{2}{*}{ Isolates } & \multicolumn{2}{|c|}{ DM 0} & \multicolumn{6}{|c|}{ Group 1} & \multicolumn{6}{|c|}{ Group 2} & \multicolumn{6}{|c|}{ Group 3} & 4 & \\
\hline & \multicolumn{21}{|c|}{ Resistance - or susceptibility + response } & \\
\hline SP Rib 1 & + & + & + & + & + & + & + & + & + & + & + & + & + & + & + & - & - & - & - & + & - & $63 / 63 / 33 / 00$ \\
\hline SP Rib 2 & + & + & + & + & + & + & + & + & + & + & + & + & + & + & + & - & - & - & - & + & - & $63 / 63 / 33 / 00$ \\
\hline SP Rib 3 & + & + & + & + & + & + & + & + & + & + & + & + & + & + & + & + & - & - & - & - & - & $63 / 63 / 03 / 00$ \\
\hline SP Jabot 1 & + & + & + & + & + & + & + & + & + & + & + & + & + & + & + & - & - & - & - & + & - & $63 / 63 / 33 / 00$ \\
\hline SP Jabot 2 & + & + & + & + & + & + & + & + & + & + & + & + & + & + & + & + & - & - & + & + & - & $63 / 63 / 51 / 00$ \\
\hline SP Jabot 3 & + & + & + & + & + & + & + & + & + & + & + & + & + & + & + & - & - & - & - & + & - & $63 / 63 / 33 / 00$ \\
\hline SP Jabot 4 & + & + & + & + & + & + & + & + & + & + & + & + & + & + & + & - & - & - & - & + & - & $63 / 63 / 33 / 00$ \\
\hline SP Bauru 1 & + & + & + & + & + & + & + & + & + & + & + & + & + & + & + & + & - & - & + & - & - & $63 / 63 / 19 / 00$ \\
\hline SP Bauru 2 & + & + & + & + & + & + & + & + & + & + & + & + & + & + & + & + & - & - & - & - & - & $63 / 63 / 03 / 00$ \\
\hline SP Bauru 3 & + & + & + & + & + & + & + & + & + & + & + & + & + & + & + & + & - & - & + & - & - & $63 / 63 / 19 / 00$ \\
\hline SP Bauru 4 & + & + & + & + & + & + & + & + & + & + & + & + & + & + & + & + & - & - & + & + & - & $63 / 63 / 51 / 00$ \\
\hline SP Bauru 5 & + & + & + & + & + & + & + & + & + & + & + & + & + & + & + & + & - & - & - & - & - & $63 / 63 / 03 / 00$ \\
\hline SP C M & + & + & + & + & + & + & + & + & + & + & + & + & + & + & + & + & - & - & - & - & - & $63 / 63 / 03 / 00$ \\
\hline SP MM 1 & + & + & + & + & + & + & + & + & + & + & + & + & + & + & + & + & - & - & - & - & - & $63 / 63 / 03 / 00$ \\
\hline SP MM 2 & + & + & + & + & + & + & + & + & + & + & + & + & + & + & + & + & - & - & - & - & - & $63 / 63 / 03 / 00$ \\
\hline SP Itapira 1 & + & + & + & + & + & + & + & + & + & + & + & + & + & + & + & + & - & - & + & + & - & $63 / 63 / 51 / 00$ \\
\hline SP Itapira 2 & + & + & + & + & + & + & + & + & + & + & + & + & + & + & + & + & - & - & - & - & - & $63 / 63 / 03 / 00$ \\
\hline SP Itapira 3 & + & + & + & + & + & + & + & + & + & + & + & + & + & + & + & + & - & - & - & - & - & $63 / 63 / 03 / 00$ \\
\hline SP Itapira 4 & + & + & + & + & + & + & + & + & + & + & + & + & + & + & + & + & - & - & + & + & - & $63 / 63 / 51 / 00$ \\
\hline SP MC 1 & + & + & + & + & + & + & + & + & + & + & + & + & + & + & + & - & - & - & - & + & - & $63 / 63 / 33 / 00$ \\
\hline SP MC 2 & + & + & + & + & + & + & + & + & + & + & + & + & + & + & + & + & - & - & + & - & - & $63 / 63 / 19 / 00$ \\
\hline SP MC 3 & + & + & + & + & + & + & + & + & + & + & + & + & + & + & + & + & - & - & - & - & - & $63 / 63 / 03 / 00$ \\
\hline SP MC 4 & + & + & + & + & + & + & + & + & + & + & + & + & + & + & + & + & - & - & - & - & - & $63 / 63 / 03 / 00$ \\
\hline SP MC 5 & + & + & + & + & + & + & + & + & + & + & + & + & + & + & + & + & - & - & - & - & - & $63 / 63 / 03 / 00$ \\
\hline SP MC 6 & + & + & + & + & + & + & + & + & + & + & + & + & + & + & + & + & - & - & + & + & - & $63 / 63 / 51 / 00$ \\
\hline SP BM 1 & + & + & + & + & + & + & + & + & + & + & + & + & + & + & + & + & - & - & - & - & - & $63 / 63 / 03 / 00$ \\
\hline SP BM 2 & + & + & + & + & + & + & + & + & + & + & + & + & + & + & + & + & - & - & - & - & - & $63 / 63 / 03 / 00$ \\
\hline SP BM 3 & + & + & + & + & + & + & + & + & + & + & + & + & + & + & + & - & - & - & - & + & - & $63 / 63 / 33 / 00$ \\
\hline SP BM 4 & + & + & + & + & + & + & + & + & + & + & + & + & + & + & + & + & - & - & - & - & - & $63 / 63 / 03 / 00$ \\
\hline SP BM 5 & + & + & + & + & + & + & + & + & + & + & + & + & + & + & + & - & - & - & - & + & - & $63 / 63 / 33 / 00$ \\
\hline SP Bot 1 & + & + & + & + & + & + & + & + & + & + & + & + & + & + & + & - & - & - & - & + & - & $63 / 63 / 33 / 00$ \\
\hline SP Bot 2 & + & + & + & + & + & + & + & + & + & + & + & + & + & + & + & + & - & - & + & - & - & $63 / 63 / 19 / 00$ \\
\hline SP Bot 3 & + & + & + & + & + & + & + & + & + & + & + & + & + & + & + & - & - & - & - & + & - & $63 / 63 / 33 / 00$ \\
\hline SP Bot 4 & + & + & + & + & + & + & + & + & + & + & + & + & + & + & + & + & - & - & - & - & - & $63 / 63 / 03 / 00$ \\
\hline SP Bot 5 & + & + & + & + & + & + & + & + & + & + & + & + & + & + & + & + & - & - & - & - & - & $63 / 63 / 03 / 00$ \\
\hline SPB1:01 & + & + & $(+)$ & $(+)$ & $(+)$ & + & $(+)$ & + & + & + & + & + & $(+)$ & $(+)$ & + & + & - & - & + & + & $(-)$ & $63 / 63 / 51 / 00$ \\
\hline SPB1:03 & + & + & + & + & + & + & + & + & + & + & + & + & $(+)$ & + & $(+)$ & $(+)$ & $(-)$ & $(-)$ & + & $(-)$ & $(-)$ & $63 / 63 / 19 / 00$ \\
\hline SPBl:04 & + & + & + & + & + & + & + & $(+)$ & + & $(+)$ & + & + & $(+)$ & $(+)$ & $(+)$ & $(+)$ & $(-)$ & $(-)$ & $(-)$ & $(-)$ & $(-)$ & $63 / 63 / 03 / 00$ \\
\hline SPBl:05 & + & + & + & + & + & + & + & + & + & + & + & + & + & + & + & - & - & - & - & + & - & $63 / 63 / 33 / 00$ \\
\hline
\end{tabular}

Ribeirão Preto (SP Rib), Jaboticabal (SP Jabot), Bauru (SP Bauru), Cândido Mota (SP CM), Mogi Mirim (SP MM), Itapira (SP Itapira),

Mogi das Cruzes (SP MC), Biritiba Mirim (SP BM), Botucatu (SP Bot). 
Table 2. Resistance/susceptibility response of lettuce differentiating cultivars to Bremia lactucae isolates collected during 2009 in the major lettuce producing regions of São Paulo state (respostas de resistência/suscetibilidade de cultivares diferenciadoras de alface a isolados de Bremia lactucae, coletados durante 2009 na maior região produtora de alface do estado de São Paulo). Jaboticabal, UNESP, 2011.

\begin{tabular}{|c|c|c|c|c|c|c|c|c|c|c|c|c|c|c|c|c|c|c|c|c|c|c|}
\hline 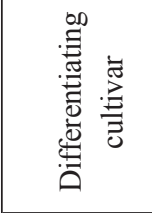 & 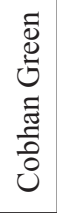 & 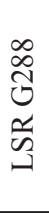 & 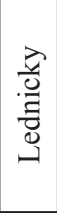 & $\begin{array}{l}\mathcal{Z} \\
\mathcal{\partial} \\
\mathcal{\partial}\end{array}$ & 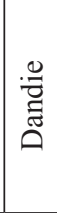 & $\begin{array}{l}\stackrel{\theta}{i n} \\
\stackrel{+}{*}\end{array}$ & 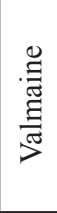 & 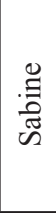 & 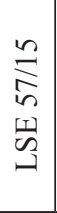 & 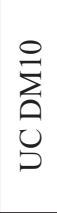 & : & 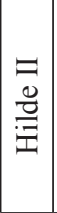 & $\mid \begin{array}{c}\frac{0}{\pi} \\
\frac{\pi}{J} \\
\frac{0}{0} \\
2\end{array}$ & 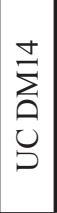 & 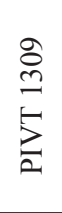 & $\begin{array}{l}0 \\
1 \\
\mathfrak{1} \\
0 \\
0 \\
0\end{array}$ & 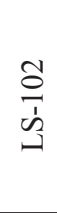 & $\begin{array}{l}\frac{0}{0} \\
\frac{\pi}{0} \\
0\end{array}$ & $\stackrel{\cdot \widetilde{\Xi}}{\Xi}$ & 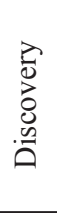 & $\begin{array}{l}\frac{u}{d} \\
\frac{\infty}{2} \\
\dot{q}\end{array}$ & \multirow{6}{*}{ 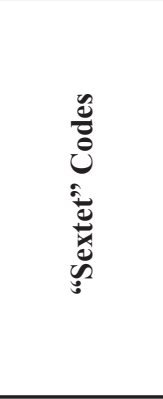 } \\
\hline Genes DM & - & - & 1 & 2 & 3 & 4 & $5 / 8$ & 6 & 7 & 10 & 11 & 12 & 13 & 14 & 15 & 16 & 17 & 18 & 36 & 37 & 38 & \\
\hline Sextet No. & - & - & 1 & 2 & 3 & 4 & 5 & 6 & 7 & 8 & 9 & 10 & 11 & 12 & 13 & 14 & 15 & 16 & 17 & 18 & 19 & \\
\hline Values & - & - & 1 & 2 & 4 & 8 & 16 & 32 & 1 & 2 & 4 & \begin{tabular}{|l|}
8 \\
\end{tabular} & \begin{tabular}{|l|}
16 \\
\end{tabular} & 32 & 1 & 2 & 4 & 8 & 16 & 32 & 1 & \\
\hline \multirow[t]{2}{*}{\begin{tabular}{|l|} 
Isolates \\
\end{tabular}} & \multicolumn{2}{|c|}{ DM 0} & \multicolumn{6}{|c|}{ Group 1} & \multicolumn{6}{|c|}{ Group 2} & \multicolumn{6}{|c|}{ Group 3} & 4 & \\
\hline & \multicolumn{21}{|c|}{ Resistance - or susceptibility + response } & \\
\hline SP Rib 1 & + & + & + & + & + & $(-)$ & $(+)$ & $(+)$ & $(+)$ & $(+)$ & + & + & + & + & $(+)$ & $(+)$ & - & - & + & - & - & $63 / 63 / 19 / 00$ \\
\hline SP Rib 2 & + & + & + & + & + & + & + & + & + & + & + & $(+)$ & $(+)$ & $(+)$ & + & $(+)$ & - & - & $(+)$ & - & $(-)$ & $63 / 63 / 19 / 00$ \\
\hline SP Rib 3 & + & + & $(+)$ & $(+)$ & $(+)$ & $(+)$ & $(+)$ & $(+)$ & $(+)$ & $(+)$ & + & + & + & + & + & $(+)$ & - & $(-)$ & + & - & - & $63 / 63 / 19 / 00$ \\
\hline SP Rib 4 & + & + & $(+)$ & $(+)$ & $(+)$ & $(+)$ & $(+)$ & $(+)$ & $(+)$ & $(+)$ & $(+)$ & $(+)$ & $(+)$ & $(+)$ & $(+)$ & + & - & - & - & $(-)$ & $(-)$ & $63 / 63 / 03 / 00$ \\
\hline SP Rib 5 & + & + & $(+)$ & $(+)$ & $(+)$ & $(+)$ & $(+)$ & $(+)$ & + & + & $(+)$ & $(+)$ & $(+)$ & $(+)$ & $(+)$ & + & - & - & $(+)$ & $(-)$ & - & $63 / 63 / 19 / 00$ \\
\hline SP Pir 1 & + & + & $(+)$ & $(+)$ & $(+)$ & $(+)$ & + & $(+)$ & + & $(+)$ & $(+)$ & $(+)$ & $(+)$ & $(+)$ & $(+)$ & $(+)$ & - & - & + & $(-)$ & $(-)$ & $63 / 63 / 19 / 00$ \\
\hline SP Pir 2 & + & + & + & + & $(+)$ & $(+)$ & $(+)$ & + & + & + & $(+)$ & $(+)$ & + & + & $(+)$ & $(+)$ & - & - & + & + & - & $63 / 63 / 51 / 00$ \\
\hline SP Pir 3 & + & + & + & $(+)$ & + & + & + & + & + & + & $(+)$ & $(+)$ & + & + & + & + & - & - & + & - & - & $63 / 63 / 19 / 00$ \\
\hline SP Cat 1 & + & + & + & + & + & + & + & + & + & + & $(+)$ & $(+)$ & + & $(+)$ & + & + & - & - & $(+)$ & - & - & $63 / 63 / 19 / 00$ \\
\hline SP Cat 2 & + & + & $(+)$ & $(+)$ & + & $(+)$ & $(+)$ & $(+)$ & + & + & $(+)$ & $(+)$ & $(+)$ & $(+)$ & + & + & - & - & - & - & - & $63 / 63 / 03 / 00$ \\
\hline SP SJRP 1 & + & + & + & + & + & + & + & + & + & $(+)$ & $(+)$ & + & + & + & + & + & - & - & - & - & - & $3 / 63 / 03 / 00$ \\
\hline SP SJRP 2 & + & + & $(+)$ & $(+)$ & + & $(+)$ & + & $(+)$ & $(+)$ & + & $(+)$ & + & + & $(+)$ & + & + & - & - & + & $(-)$ & $(-)$ & $3 / 63 / 19 / 00$ \\
\hline SPSJRP 3 & + & + & + & $(+)$ & + & $(+)$ & $(+)$ & + & + & + & + & + & + & + & + & + & 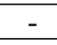 & 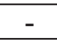 & - & - & - & $3 / 63 / 03 / 00$ \\
\hline SPSJRP 4 & + & + & + & + & + & + & + & $(+)$ & + & + & + & + & + & + & + & + & - & - & + & + & - & $3 / 63 / 51 / 00$ \\
\hline SPSJRP 5 & + & + & + & + & $(+)$ & $(+)$ & + & + & + & + & + & + & + & + & + & + & - & - & + & - & - & $3 / 63 / 19 / 00$ \\
\hline SP Atib 1 & + & + & + & $(+)$ & + & $(+)$ & + & + & + & $(+)$ & + & + & + & + & + & + & - & - & + & + & $(-)$ & $63 / 63 / 51 / 00$ \\
\hline SP Atib 2 & + & + & + & $(+)$ & + & + & + & + & + & + & + & + & + & $(+)$ & + & + & - & $(-)$ & + & + & - & $63 / 63 / 51 / 00$ \\
\hline SP Atib 3 & + & + & $(+)$ & + & + & $(+)$ & + & + & + & $(+)$ & + & + & + & $(+)$ & + & + & - & $(-)$ & + & - & - & $63 / 63 / 19 / 00$ \\
\hline SP Sal 1 & + & + & $(+)$ & $(+)$ & + & + & + & $(+)$ & + & $(+)$ & + & + & + & $(+)$ & + & + & - & - & - & $(-)$ & $(-)$ & $63 / 63 / 03 / 00$ \\
\hline SP Sal 2 & + & + & $(+)$ & $(+)$ & $(+)$ & + & + & + & + & $(+)$ & $(+)$ & $(+)$ & + & + & + & + & - & - & + & + & $(-)$ & $63 / 63 / 51 / 00$ \\
\hline SP BM 1 & + & + & $(+)$ & + & + & + & + & + & + & $(+)$ & + & + & + & $(+)$ & + & + & - & - & + & + & - & $63 / 63 / 51 / 00$ \\
\hline SP BM 2 & + & + & $(+)$ & $(+)$ & + & $(+)$ & + & + & + & + & + & + & + & $(+)$ & $(+)$ & $(+)$ & - & - & - & - & - & $3 / 63 / 03 / 00$ \\
\hline SP BM 3 & + & + & + & $(+)$ & + & + & + & + & + & + & + & $(+)$ & + & + & + & + & - & $(-)$ & + & - & - & $3 / 63 / 19 / 00$ \\
\hline SP Itap 1 & + & + & + & + & + & $(+)$ & + & $(+)$ & + & $(+)$ & $(+)$ & $(+)$ & $(+)$ & $(+)$ & $(+)$ & $(+)$ & - & - & + & $(-)$ & $(-)$ & $63 / 19 / 00$ \\
\hline SP Itap 2 & + & + & + & + & + & $(+)$ & $(+)$ & + & + & + & + & + & + & $(+)$ & $(+)$ & + & - & $(-)$ & $(-)$ & $(-)$ & - & $3 / 63 / 03 / 00$ \\
\hline SP Itap 3 & + & + & + & + & + & + & $(+)$ & + & + & + & + & $(+)$ & + & + & - & + & - & - & - & - & - & $63 / 63 / 02 / 00$ \\
\hline SP Itap 4 & + & + & + & + & $(+)$ & $(+)$ & + & + & + & - & + & + & $(+)$ & $(+)$ & $(+)$ & $(+)$ & 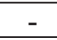 & - & + & + & - & $63 / 63 / 51 / 00$ \\
\hline SP MM 1 & + & + & $(+)$ & + & + & + & $(+)$ & $(+)$ & - & $(+)$ & $(+)$ & $(+)$ & $(+)$ & $(+)$ & $(+)$ & $(+)$ & - & - & + & + & $(-)$ & $63 / 63 / 51 / 00$ \\
\hline SP MM 2 & + & + & + & + & + & $(+)$ & $(+)$ & $(+)$ & $(+)$ & + & + & + & + & $(+)$ & $(+)$ & $(+)$ & - & - & + & + & $(-)$ & $63 / 63 / 51 / 00$ \\
\hline SP MM 3 & + & + & + & + & $(+)$ & + & + & + & + & + & + & + & + & $(+)$ & + & $(+)$ & - & - & + & - & $(-)$ & $63 / 63 / 19 / 00$ \\
\hline SP CM 1 & + & + & $(+)$ & $(+)$ & $(+)$ & $(+)$ & $(+)$ & $(+)$ & $(+)$ & + & $(+)$ & $(+)$ & + & + & $(+)$ & $(+)$ & - & - & $(-)$ & $(-)$ & $(-)$ & $3 / 63 / 03 / 00$ \\
\hline SP CM 2 & + & + & + & $(+)$ & + & + & + & + & + & + & + & + & + & + & - & $(+)$ & - & - & $(-)$ & $(-)$ & - & $63 / 63 / 02 / 00$ \\
\hline SP CM 3 & + & + & + & + & + & $(+)$ & $(+)$ & + & + & $(+)$ & + & + & $(+)$ & $(+)$ & $(+)$ & $(+)$ & $(-)$ & $(-)$ & + & + & $(-)$ & $3 / 63 / 51 / 00$ \\
\hline SPCM 4 & + & + & + & + & $(+)$ & $(+)$ & $(+)$ & $(+)$ & $(+)$ & + & + & $(+)$ & + & + & $(+)$ & $(+)$ & $(-)$ & $(-)$ & + & + & - & $3 / 63 / 51 / 00$ \\
\hline SP PP 1 & + & + & + & + & + & + & + & $(+)$ & + & $(+)$ & + & + & $(+)$ & $(+)$ & $(+)$ & $(+)$ & $(-)$ & $(-)$ & $(-)$ & $(-)$ & $(-)$ & $63 / 63 / 03 / 00$ \\
\hline SP PP 2 & + & + & $(+)$ & $(+)$ & + & + & + & + & + & + & + & + & $(+)$ & $(+)$ & $(+)$ & $(+)$ & $(-)$ & $(-)$ & $(-)$ & $(-)$ & $(-)$ & $63 / 63 / 03 / 00$ \\
\hline SPPP 3 & + & + & + & + & $(+)$ & $(+)$ & + & + & + & + & + & + & $(+)$ & $(+)$ & + & + & - & - & + & $(-)$ & $(-)$ & $63 / 63 / 19 / 00$ \\
\hline SP PP 4 & + & + & $(+)$ & $(+)$ & $(+)$ & + & $(+)$ & + & + & + & + & + & $(+)$ & $(+)$ & + & + & - & - & + & + & $(-)$ & $63 / 63 / 51 / 00$ \\
\hline SP PP 5 & + & + & + & + & + & $(+)$ & + & + & $(+)$ & + & $(+)$ & $(+)$ & + & + & + & + & - & $(-)$ & + & $(-)$ & $(-)$ & $63 / 63 / 19 / 00$ \\
\hline SP Ech 1 & + & + & $(+)$ & $(+)$ & + & + & $(+)$ & $(+)$ & + & $(+)$ & + & + & $(+)$ & $(+)$ & + & + & $(-)$ & $(-)$ & - & - & - & $63 / 63 / 03 / 00$ \\
\hline SP Mar 1 & + & + & + & + & $(+)$ & $(+)$ & + & $(+)$ & + & + & + & $(+)$ & $(+)$ & $(+)$ & $(+)$ & $(+)$ & - & - & + & $(-)$ & - & $63 / 63 / 19 / 00$ \\
\hline SP Mar 2 & + & + & $(+)$ & + & + & $(+)$ & + & + & $(+)$ & $(+)$ & $(+)$ & $(+)$ & $(+)$ & $(+)$ & + & + & - & $(-)$ & + & $(-)$ & $(-)$ & $63 / 63 / 19 / 00$ \\
\hline SP Mar 3 & + & + & $(+)$ & $(+)$ & + & + & + & + & + & $(+)$ & + & $(+)$ & + & + & $(+)$ & $(+)$ & - & - & + & $(-)$ & $(-)$ & $63 / 63 / 19 / 00$ \\
\hline SP Mar 4 & + & + & + & $(+)$ & + & + & + & + & $(+)$ & + & + & + & + & + & + & $(+)$ & $(-)$ & $(-)$ & + & $(-)$ & - & $63 / 63 / 19 / 00$ \\
\hline SP Mar 5 & + & + & + & + & $(+)$ & + & + & + & + & $(+)$ & + & + & + & + & $(+)$ & + & - & - & + & $(-)$ & $(-)$ & $63 / 63 / 19 / 00$ \\
\hline SP Bot 1 & + & + & + & + & + & + & + & + & + & + & + & + & $(+)$ & + & $(+)$ & $(+)$ & $(-)$ & $(-)$ & + & $(-)$ & $(-)$ & $63 / 63 / 19 / 00$ \\
\hline SP Bot 2 & + & + & $(+)$ & $(+)$ & $(+)$ & + & + & + & + & $(+)$ & + & + & $(+)$ & + & $(+)$ & + & $(-)$ & - & $(-)$ & $(-)$ & $(-)$ & $63 / 63 / 03 / 00$ \\
\hline SP Bauru 1 & + & + & + & + & $(+)$ & $(+)$ & + & $(+)$ & + & + & $(+)$ & $(+)$ & $(+)$ & + & $(+)$ & $(+)$ & $(-)$ & - & + & + & $(-)$ & $63 / 63 / 51 / 00$ \\
\hline SP Bauru 2 & + & + & $(+)$ & $(+)$ & $(+)$ & + & + & + & + & + & + & + & + & $(+)$ & $(+)$ & + & - & - & + & $(-)$ & $(-)$ & $63 / 63 / 19 / 00$ \\
\hline SPBI:01 & + & + & $(+)$ & $(+)$ & $(+)$ & + & $(+)$ & + & + & + & + & + & $(+)$ & $(+)$ & + & + & - & - & + & + & $(-)$ & $63 / 63 / 51 / 00$ \\
\hline SPBI:03 & + & + & + & + & + & + & + & + & + & + & + & + & $(+)$ & + & $(+)$ & $(+)$ & $(-)$ & $(-)$ & + & $(-)$ & $(-)$ & $63 / 63 / 19 / 00$ \\
\hline SPBI:04 & + & + & + & + & + & + & + & $(+)$ & + & $(+)$ & + & + & $(+)$ & $(+)$ & $(+)$ & $(+)$ & $(-)$ & $(-)$ & $(-)$ & $(-)$ & $(-)$ & $63 / 63 / 03 / 00$ \\
\hline SPBI::06 & + & + & + & $(+)$ & + & + & + & + & + & + & + & + & + & + & - & $(+)$ & - & - & $(-)$ & $(-)$ & - & $63 / 63 / 02 / 00$ \\
\hline
\end{tabular}

Ribeirão Preto (SP Rib), Pirangi (SP Pir), Catanduva (SP Cat), São José do Rio Preto (SP SJRP), Atibaia (SP Atib), Salesópolis (SP Sal), Biritiba Mirim (SP BM), Itapira (SP Itap), Mogi Mirim (SP MM), Cândido Mota (SP CM), Presidente Prudente (SP PP), Echaporã (SP Ech), Marília (SP Mar), Botucatu (SP Bot), Bauru (SP Bauru). 
The resistance, or susceptibility, of the 49 isolates collected during 2009 to the genes DM present in the lettuce differentiating cultivars are shown in Table 2. Among the isolates, four codes of $B$. lactucae (63/63/19/00, 63/63/51/00, 63/63/03/00, and $63 / 63 / 02 / 00)$ were identified. These codes were found in 44.90, 26.53, 24.49, and $4.08 \%$ of the collected samples, respectively. The new code found in $2008(63 / 63 / 33 / 00)$ was not found in any other of the isolates collected in 2009 . Differences in the selection pressure brought about by the intensive use of fungicides are supposed to explain that fact.

The data show the predominance of race SPBl:03 (63/63/19/00), and the rapid dispersion of sporangia, since this code was first identified in 2007 in a research of Souza et al. (2011).

The sextet code 63/63/51/00, frequently found in 2009 , was identified in 2003 and 2004 in a pioneer study of Bremia lactucae in Brazil (Braz et al., 2007) and nominated SPB1:01. This race was found to be less frequent in subsequent years (2006 and 2007), probably as a consequence of the appearance of new races. This fact is interpreted as an indication that the monitoring of lettuce producing areas is highly important since new pathogen's genes, capable of breaking down of the resistance of the lettuce cultivars, are likely to be introduced or result from mutations.

The evaluation of the results by code and the comparison with those already existing in Brazil (63/63/51/00, $63 / 31 / 19 / 00,63 / 63 / 19 / 00$, and $63 / 63 / 19 / 00$ ) show that the sextet code $63 / 63 / 02 / 00$ was first found in 2009 and this resulted in it being nominated SPB1:06. Though not occurring predominantly (it was identified in only $4.08 \%$ of the isolates), this race deserves special attention due to its rapid dispersion thus having a high potential to cause severe financial losses to lettuce farmers.

The genes conferring resistance to this new race are: DM-15, R-17, R-18, $\mathrm{R}-36, \mathrm{R}-37$, and R-38. The resistance of the gene DM-15 to this race is a distinguishing character when it is compared to the races identified till now. But, this gene should not be used in breeding programs due to its sensibility to the codes $63 / 63 / 51 / 00,63 / 31 / 19 / 00$, $63 / 63 / 19 / 00$, and 63/63/03/00.

The data herein presented are showing that two new codes have appeared in São Paulo state: 63/63/33/00 and $63 / 63 / 02 / 00$ respectively denominated SPB1:05 and SPB1:06. The genes R-17, R-18 and R-38 are recommended to be introduced in lettuce cultivars resulting from breeding programs not only because they confer resistance to the B. lactucae races found by Braz et al. (2007) and Souza et al. (2011) but also to those mentioned in this paper, that is, races $\mathrm{SPBl}: 05$ and $\mathrm{SPBl}: 06$.

However, it is suggested that the identification of races will be annual, since these change is constant, because of the selection pressure caused by constant use of herbicides, environmental changes and use of vertical resistance, among other factors.

\section{ACKNOWLEDGEMENTS}

The authors thank the financial contributions given by FAPESP (Research Funding Agency of the Government of the State of São Paulo) and by CNPq (National Council for the Scientific and Technological Development) to do this investigation.

\section{REFERENCES}

AGRIANUAL 2011: Anuário da agricultura brasileira. 2011. São Paulo: FNP Consultoria e Agroinformativo. p. 135.

BRAZ LT; DALPIAN T; PISSARDI MA. 2007. Identification of races of Bremia lactucae in São Paulo, Brazil. Acta Horticulturae 760: 317-321.

IEA - Instituto de Economia Agrícola. 2010, 23 de novembro. Área e produção dos principais produtos da agropecuária. Disponível em http://ciagri.iea.sp.gov.br/bancoiea/subjetiva. aspx ?cod_sis $=1 \&$ idioma $=1$.

ILOTT TW; DURGAN ME, MICHELMORE RW. 1987. Genetics of virulence in California populations of Bremia lactucae (Lettuce Downy Mildew). Phytopathology 77: 13811386.

MICHELMORE RW; INGRAM DS. 1982. Secondary homothalism in Bremia lactucae. Transactions of the British Mycological Society 78: 1-9.

OLIVEIRAACB; SEDYIAMA MAN; PEDROSA MW; GARCIA NCP; GARCIA SLR. 2004. Divergência genética e descarte de variáveis em alface cultivada sob sistema hidropônico. Acta Scientiarum Agronomy 26: 211-217.

PLANTUM NL. 2010, 06 de novembro. New race of Bremia lactucae Bl: 27 identified and nominated. Disponível em: http://www. plantum.nl/plantum/persberichten/10-05-03. pdf.

SCHETTINI TM; LEGG EJ; MICHELMORE RW. 1991. Insensitivity to metalaxyl in California populations of Bremia lactucae and resistance of California lettuce cultivars to downy mildew. Phytopathology 81: 64-70.

SOUZA JO; DALPIAN T; BRAZ LT; CAMARGO M. 2011. Três novas raças de Bremia lactucae, agente causador de míldio da alface, identificadas no Estado de São Paulo. Jaboticabal: UNESP-FCAV. Horticultura Brasileira 29: 282-286.

VAN BRUGGEN AHC; SCHERM H. 1997. Compendium of lettuce diseases. In: DAVIS RM; SUBBARAO KV; RAID RN; KURTS EA. Down mildew. Saint Paul: American Phytopathological Society. p. 17-19.

VAN DER AREND AJM; GAUTIER J; GUENARD M; MICHEL H; MOREAU B; DE RUIJTER J; SCHUT JW; WITTE I. 2003. Identification and denomination of 'new' races of Bremia lactucae in Europe by IBEB until 2002. In: HINTUM TJL; VAN LA; PINK D; SCHUT JW (eds). Eucarpia Leafy Vegetables 2003. Wageningen: Center for Genetic Resources the Netherlands (CGN). p. 151.

YURI JE; RESENDE GM; MOTA JH; SOUZA RJ; RODRIGUES JÚNIOR JC. 2004. Comportamento de cultivares e linhagens de alface americana em Santana da Vargem (MG), nas condições de inverno. Horticultura Brasileira 22: 322-325. 